\title{
A Novel Classification and Staging System to Address Prognosis and Treatment Decisions for Patients with Granular Myringitis
}

\author{
Mohan Bansal ${ }^{1}$ \\ ${ }^{1}$ C. U. Shah Medical College, Surendranagar, Gujarat, India
}

Ann Otol Neurotol ISO 2018;1:77-82

\begin{abstract}
Address for correspondence Mohan Bansal, MS, PhD, FICS, FACS, GPO Road, Old ST Stand, Anand 388001, Gujarat, India (e-mail: mohanbansal@yahoo.com).
\end{abstract}

\begin{abstract}
Keywords

- granular myringitis

- tympanic membrane

- external auditory canal

- myringitis

- otitis

- classification

- staging

- review

Objective Does the severity of disease influence outcomes in patients with granular myringitis (GM)? There is no classification and staging system on the severity of GM although it is not a very rare disorder. The aim of this study was to propose a novel classification and staging system for the patients with GM according to the type, extent, and sequelae of the lesions so that indications of various modalities of treatments for the different classes of the disease and their outcomes could be studied.

Methods Data were retrieved from PubMed, Embase, and Cochrane Library. Relevant articles were also sought by a hand search review of references, books, and journals. The databases were searched using the keywords "granular myringitis," "granular otitis externa," "myringitis granulosa," and "myringitis." Data from the relevant articles were extracted using a pre-defined data-extraction form.

Results After reviewing the various otoscopic findings, the study proposes a clinical and surgical GAS classification and staging of granular myringitis, which is based on

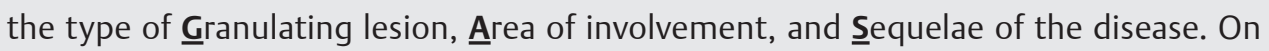
the basis of GAS classification, patients with GM are grouped into four stages that indicate the overall severity and prognosis of the disease and can be used for selecting various modalities of treatment and their outcomes.

Conclusion The GAS classification is simple, descriptive, and comprehensive and would provide practical basis for the indications of different modalities of management and outcomes research in patients with GM. This would facilitate in knowing the prognostic impact of extent of granulations.

This study has a 1a type level of evidence.
\end{abstract}

\section{Introduction}

Granular myringitis (GM) is an idiopathic inflammatory process of tympanic membrane (TM) characterized by the granulation tissue and mucosalized epithelium of the $\mathrm{TM} ;{ }^{1}$ in advanced cases, TM may get thickened. ${ }^{2}$ Various modalities of treatment have been employed in the management of this disorder. ${ }^{3} \mathrm{GM}$ is not rare ${ }^{4}$ and has various types of lesions, which vary in extension and can have important bearing on the line of treatment and prognosis. It can affect not only TM but can also extend to deep bony external auditory canal (EAC). ${ }^{5,6}$ The prevalence of GM was found to be approximately one fourth as common as cholesteatoma. ${ }^{7}$ GM can involve different layers of TM and can even lead to its perforation. ${ }^{4,7,8}$ Medical and surgical intervention is varied and often does not lead to permanent resolution of the disease..$^{3,9}$ Untreated GM can lead to inflammatory infiltration of the deep EAC and post-inflammatory medial EAC fibrosis and its atresia. ${ }^{10}$

In spite of all these varied otoscopy findings, sequelae (causing hearing impairment), and diverse treatments, 
currently there is no classification and staging system of GM. The clinical and surgical classification helps in not only describing distinct disease patterns and guide management but also facilitates outcomes research. The oncological TNM classification and staging is the best example.

Patients with GM have considerable variations in lesions. The present study investigates the variations in lesions of GM and proposes a novel classification and staging system, which is based on the type and extent of lesions and the sequelae. The classification will provide an idea about the severity of the disease and aid in decision making regarding the various modalities of treatment and their outcomes.

\section{Methods}

This study was granted an exception from ethics approval by the Institutional Ethics Committee.

\section{Data Sources}

The literature and data sources were explored in a thorough and comprehensive manner with the assistance of the librarian (PN). This article included studies published in English that examined the otoscopy findings related with the type and extent of lesions and sequelae of the primary GM. The databases searched were PubMed, EMBASE, and the Cochrane Library. No time limit was set and databases were searched from time of their inception. The search was completed by June 2015. The databases were searched using Boolean combinations of the keywords "granular myringitis," "granular otitis externa," "myringitis granulosa," and "myringitis" in title and abstract fields. No limits were applied; however, author did exclude publications that did not have abstracts. Author evaluated the titles and abstracts of all the studies identified in the initial search to locate any potentially relevant studies. The full texts of studies identified as potentially relevant were then evaluated. These were augmented by their references and option of similar articles. Relevant articles were also sought by a hand search review of references, books, and journals.

\section{Inclusion and Exclusion Criteria}

Studies were eligible if they met the following criteria: studies that examined the otoscopic findings related with the type and extent of lesion and sequelae of primary GM. The studies of secondary $\mathrm{GM}^{10}$ associated with diseases and surgeries of EAC and middle ear were not included in the study. The acute, hemorrhagic, and bullous myringitis studies were excluded. In cases of multiple publications of the same or overlapping cohorts, only the studies with the largest sample size were included. The following types of studies were excluded: reviews, commentaries, case reports or letters, and studies with insufficient reported data.

\section{Data Extraction and Level of Evidence}

Data from the articles were extracted using a pre-defined data-extraction form. Discrepancies were noted and discussed between the two other faculties (N.H. and C.R.) in the E.N.T. department and resolved by consensus. The following data were recorded for each study: (1) year of publication; (2) country;
(3) study design; (4) number of cases; (5) findings of otoscopy/ ear-micro-examination/otoendoscopy, and (6) treatment. Levels of evidence were ascertained for each study. Disagreements were resolved by discussions with the biostatistician (A.P.).

\section{Results}

\section{Trial Selection}

The initial search strategy (excluding duplicates) retrieved 122 publications. After screening all titles and abstracts, the search strategy retrieved 28 publications for possible inclusion in the systematic review. Among these 28 publications, nine publications met the inclusion criteria and were retrieved for more in-depth evaluation (-Fig. 1).

\section{Description of Studies}

These nine studies from 2000 through 2011 (-Table 1) reported otoscopic findings related to the type and extent of lesion and sequelae of primary GM. Two of the nine studies were conducted in Taiwan, ${ }^{11,12}$ two in Israel, ${ }^{4,13}$ two in Egypt, ${ }^{6,8}$ one in Korea, ${ }^{5}$ one in United States, ${ }^{7}$ and one in China. ${ }^{14}$ Levels of evidence were ascertained for each study. Only one of the nine studies was controlled double-blinded ${ }^{6}$; two were prospective ${ }^{4,13}$ and remaining six were retrospective., ${ }^{5,7,11,12,14}$

These nine clinical studies included 335 patients with GM (-Table 1). One study included only children as their subjects. ${ }^{13}$ All studies were published in English. Because of the heterogeneity in study design, data collection, and data presentation, I did not pool summary data in a meta-analysis. There were particular weaknesses in terms of outcome ascertainment and the representative nature of the cases.

\section{Study Findings}

All the studies found de-epithelization and/or granulations on TM in their patients with GM (-Table 1). Three studies found polypoidal formation ${ }^{4,5,13}$ and four studies had TM perforation. ${ }^{4,7,8,13}$ The EAC was found involved in three studies $6,7,14$ and in remaining six studies lesions were limited to only TM (-Table 1). One study even mentioned about the acquired atresia of EAC. ${ }^{7}$ The terms used for the extent of TM lesions were focal, ${ }^{2}$ circumscribed, ${ }^{13}$ patchy, ${ }^{8}$ segmental, ${ }^{8,12}$ diffuse, ${ }^{8,12,14}$ and entire TM. ${ }^{4}$ Acute granulomatous myringitis ${ }^{15}$ cases were not included. The GM was also termed as myringitis granulosa. ${ }^{16}$

Four clinical studies $5,7,8,13$ employed both surgical and topical treatments while two studies had only topical medical therapy, ${ }^{4,6}$ and in three studies mainly surgical treatment ${ }^{11,12,14}$ was used. The surgical modalities of treatment included $\mathrm{CO}_{2}$ laser, ${ }^{11,12,17}$ mechanical removal of granulations, $5,7,8,13$ epithelial avulsion with overlay myringoplasty. ${ }^{14}$ The topical medical treatment included antibiotic ear drops, ${ }^{4,7,8,13}$ Castellani solution, ${ }^{5} 5$-fluorouracil 5\% cream, ${ }^{6}$ vinegar, ${ }^{18}$ formalin, ${ }^{19}$ and other caustic agents. ${ }^{413}$ No clinical and surgical classification and staging of GM was found.

\section{Proposed Clinical and Surgical GAS Classification and Staging for Granular Myringitis}

The study has devised a comprehensive four-tier classification scheme with the primary goal of stratifying lesions 


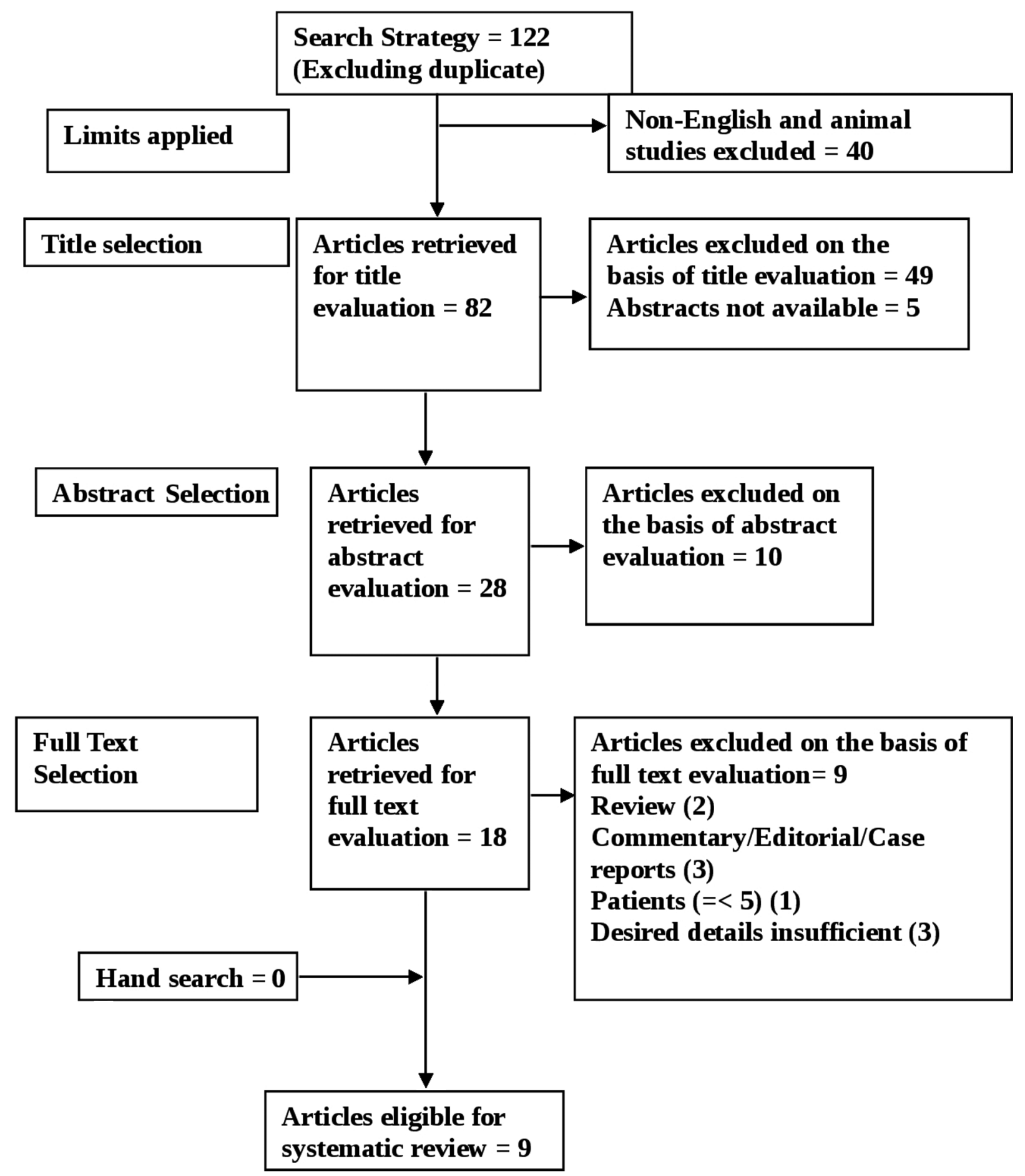

Fig. 1 Flow chart depicting the method of study selection.

based on type, extent, and sequelae of GM. On the basis of the otoscopic findings, I propose the following $\mathbf{G}$ A S classification that takes into consideration the type of $\underline{\text { Granulating }}$

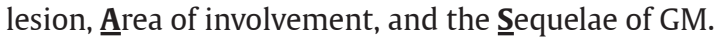

1. Types of $\underline{G}$ ranulating lesion $\left(G_{1}, G_{2}, G_{3}\right.$ and $\left.G_{4}\right)$
a. $\mathrm{G}_{1}$ : Only de-epithelization
b. $G_{2}$ : Sessile granulations
c. $\mathrm{G}_{3}$ : Pedunculated granulations
d. $G_{4}$ : Polyp formations

2. Area of involvement $\left(A_{1}, A_{2}, A_{3}\right.$ and $\left.A_{4}\right)$

a. $A_{1}$ : Single discrete lesion involving not more than quarter of TM

b. $A_{2}$ : Single or multiple lesions involving quarter to half of the TM

c. $A_{3}$ : Single or multiple lesions involving more than half of the TM but not involving deep bony EAC.

d. $A_{4}$ : Lesions present and/or extending into the deep bony EAC 
Table 1 Summary of the nine studies that investigated the clinical findings in patients with GM

\begin{tabular}{|c|c|c|c|c|}
\hline Study & Cases & Otoscopy; Ear endoscopy/microscopy & Treatment & Level of evidence \\
\hline $\begin{array}{l}\text { Kim } 2011 \text { Korea }^{3} \\
\text { Retrospective }\end{array}$ & 24 & $\begin{array}{l}\text { Marginal/non-marginal type and deepi- } \\
\text { thelialized (ulcerative)/polypoid subtype } \\
\text { using a new scale. }\end{array}$ & $\begin{array}{l}\text { Castellani solution, } \\
\text { curettage }\end{array}$ & $2 b$ \\
\hline $\begin{array}{l}\text { Zhang et al,2010 } \\
\text { China }{ }^{12} \\
\text { Retrospective }\end{array}$ & 21 & $\begin{array}{l}\text { Diffuse granulation tissue over TM, with } \\
\text { or without involvement of EAC }\end{array}$ & $\begin{array}{l}\text { Epithelial avulsion with } \\
\text { overlay myringoplasty }\end{array}$ & $2 c$ \\
\hline $\begin{array}{l}\text { Atef et al } 2010 \text { Egypt }^{4} \\
\text { Controlled, } \\
\text { double-blinded }\end{array}$ & 60 & $\begin{array}{l}\text { Granulation tissue over TM with or } \\
\text { without inflammatory obliteration of } \\
\text { deep EAC }\end{array}$ & $\begin{array}{l}\text { Topical 5-fluorouracil } \\
5 \% \text { cream }\end{array}$ & $1 \mathrm{~b}$ \\
\hline $\begin{array}{l}\text { Cheng \& Shiao } 2008 \\
\text { Taiwan }^{9} \\
\text { Retrospective }\end{array}$ & 30 & Nonepithelialized TM & $\mathrm{CO}_{2}$ laser & $2 c$ \\
\hline $\begin{array}{l}\text { Wolf et al } 2006 \text { Israel }^{2} \\
\text { Prospective }\end{array}$ & 31 & $\begin{array}{l}\text { Focal de-epithelization, focal polypoid } \\
\text { granulations, diffuse polypoid formation } \\
\text { over entire TM, perforation of TM }\end{array}$ & $\begin{array}{l}\text { Topical application of } \\
\text { antibiotics or caustic } \\
\text { agents }\end{array}$ & $2 b$ \\
\hline $\begin{array}{l}\text { Jang et al } 2006 \\
\text { Taiwan }^{10} \\
\text { Retrospective }\end{array}$ & 21 & $\begin{array}{l}\text { Segmental and diffuse granulation tissue } \\
\text { over TM }\end{array}$ & $\mathrm{CO}_{2}$ laser therapy & $2 c$ \\
\hline $\begin{array}{l}\text { Blevins \& Karmody } \\
2001 \text { USA }^{5} \\
\text { Retrospective }\end{array}$ & 45 & $\begin{array}{l}\text { Granulation tissue and perforation of } \\
\text { TM, acquired atresia of EAC }\end{array}$ & $\begin{array}{l}\text { Topical medications } \\
\text { and surgery }\end{array}$ & 4 \\
\hline $\begin{array}{l}\text { Wolf et al } 2001 \\
\text { Israel }^{11} \\
\text { Prospective }\end{array}$ & 9 & $\begin{array}{l}\text { De-epithelization, granulations over cir- } \\
\text { cumscribed area of TM, as a tiny shallow } \\
\text { lesion or raised polypoidal masses; small } \\
\text { perforation of TM }\end{array}$ & $\begin{array}{l}\text { Ear drops, caustic } \\
\text { solution, mechanical } \\
\text { removal }\end{array}$ & $2 b$ \\
\hline $\begin{array}{l}\text { El-Seifi \& Fouad } 2000 \\
\text { Egypt }^{6} \\
\text { Retrospective }\end{array}$ & 94 & $\begin{array}{l}\text { Granular areas, patchy, diffuse, or seg- } \\
\text { mental; TM perforation }\end{array}$ & $\begin{array}{l}\text { Conservative and } \\
\text { surgical }\end{array}$ & $2 c$ \\
\hline
\end{tabular}

Abbreviations: EAC, external auditory canal; TM, tympanic membrane.

3. Sequelae of lesion $\left(\mathrm{S}_{1}, \mathrm{~S}_{2}, \mathrm{~S}_{3}\right.$, and $\left.\mathrm{S}_{4}\right)$

a. $\mathrm{S}_{1}$ : Just involvement of only cuticular layer of TM (No sequelae)

b. $\mathrm{S}_{2}$ : Involvement of cuticular and fibrous layer and/or thickening of TM

c. $\mathrm{S}_{3}$ : Perforation of TM

d. $\mathrm{S}_{4}$ : Atresia of deep bony EAC

On the basis of above otoscopic GAS findings, I grouped the patients with GM into the following four stages, which indicate the overall severity and prognosis of the disease.

1. Stage I: (I-a, I-b and I-c)-GM-I
a. $G_{1}, A_{1}, S_{1}$
b. $G_{2}, A_{1}, S_{1}$
c. $G_{3}, A_{1}, S_{1}$

2. Stage II: (II-a, II-b and II-c)-GM-II
a. $G_{1-3}, A_{2}, S_{1}$
b. $G_{1-3}, A_{1}, S_{2}$
c. $\mathrm{G}_{1-3}, \mathrm{~A}_{2}, \mathrm{~S}_{2}$

3. Stage III: (III-a, III-b and III-c)-GM-III
a. $G_{1-3}, A_{3}, S_{1-2}$
b. $G_{1-3}, A_{1-2}, S_{3}$ (GM with TM perforation)
c. $G_{1-3}, A_{3}, S_{3}$ (GM with TM perforation)

4. Stage IV: (IV-a, IV-b and IV-c)-GM-IV
a. $G_{4}, A_{1-3}, S_{1-3}$ (GM with ear polyp)
b. $G_{1-4}, A_{4}, S_{1-3}$ (GM with granular otitis externa)
c. $\mathrm{G}_{1-4}, \mathrm{~A}_{1-4}, \mathrm{~S}_{4}$ (GM with stenosis of EAC)

\section{Discussion}

This study has presented a simple comprehensive classification and staging system for GM)that would be able to assist in the prognosis and decision making for the patients with GM. GM is a disease that is poorly understood and has received relatively little attention in the literature. ${ }^{20} \mathrm{GM}$ is not a rare pathologic condition of the TM. ${ }^{21} \mathrm{GM}$ has a prolonged chronic course and is characterized by de-epithelization/granulation tissue on the lateral surface of TM, which over a period of months or years may progress to TM perforation and spread laterally along the deep bony EAC. Over a period of one to three decades, squamous epithelium might grow over granulation tissue and give rise to thickened TM and/or stenosis of bony EAC.

The proposed GAS classification and staging of GM is based upon the type of $\underline{G}$ ranulating lesion, $\underline{\text { Area of involve- }}$

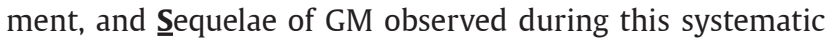
review study: 
(1) Granulating lesion: Granulations/de-epithelization (-Table 1); polypoidal formation. ${ }^{45,13}$ Instead of deepithelization some also use the term mucosalization. ${ }^{1,2}$

(2) Area of involvement: Focal, ${ }^{4}$ circumscribed, ${ }^{13}$ patchy, ${ }^{8}$ segmental, ${ }^{8,12}$ diffuse, ${ }^{8,12,14}$ and entire ${ }^{4}$ involvement of TM; and involvement of EAC. $6,7,14$

(3) Sequelae of the disease: Thickening of $\mathrm{TM}^{1}$ and its perforation ${ }^{4,7,8,13}$ and atresia of EAC. ${ }^{7}$

$G_{1}, G_{2}, G_{3}$ and $G_{4}$ types of $\mathbf{G r a n u l a t i n g ~ l e s i o n ~ d e s i g n a t e ~ d e - e p - ~}$ ithelization, sessile granulations, pedunculated granulations and polyp formation respectively. Hence, the mildest $G\left(G_{1}\right)$ is just de-epithelization of the lateral surface of TM while most severe $G\left(G_{4}\right)$ is frank polyp formation. Patients in whom multiple types of lesions are present (such as de-epithelization, sessile and pedunculated granulations and/or polyp), should mention all the types of $\mathrm{G}$, but for staging purpose, most severe form present is considered. As the active stage of GM must be distinguished from chronic suppurative otitis media (CSOM), ear-micro-examination is advised before the GAS classification. Granulations are usually covered with purulent secretions or dry crust. The aural toilet under microscope reveals whether the granulations are localized or diffuse/sessile or pedunculated. The granulations usually have a sessile base and may be pedunculated. One or more polyps may be present in some cases. ${ }^{13}$

$A_{1}, A_{2}, A_{3}$ and $A_{4}$ Area of involvement refer lesion involving not more than quarter of TM, quarter to half of the TM, more than half of the TM but not involving deep bony EAC and present and/or extending to deep bony EAC respectively. Hence, the minimum $A\left(A_{1}\right)$ is most limited lesion, whereas $A_{4}$ is the most extensive lesion. The untreated GM was found to cause inflammatory infiltration of the deep EAC., ${ }^{610}$ The granular areas may be patchy, diffuse, or segmental. The latter is the most frequent and is most commonly posterosuperior. ${ }^{8}$ In diffuse form, a slightly raised carpet of granulations covers the TM. One prospective study ${ }^{13}$ classified otoscopic findings into three grades: focal de-epithelization, focal polypoid granulations, and diffuse polypoid formation over the entire TM..$^{13}$ In cases of GM without perforation of TM, there would be normal movement of the TM on pneumatic otoscopy.

The $\mathrm{S}_{1}, \mathrm{~S}_{2}, \mathrm{~S}_{3}$ and $\mathrm{S}_{4}$ Sequelae of GM indicate involvement of only cuticular layer, cuticular and fibrous layer, and/or thickening, and perforation of TM, and atresia of deep bony EAC, respectively. Hence, in $S_{1}$, there is no sequelae as the GM is limited to epithelial layer of $\mathrm{TM}$ and $\mathrm{S}_{4}$ designates the most severe sequela atresia of deep bony EAC. $S_{3}$ is perforation of TM. Patients with GM with $\mathrm{S}_{2}$ sequela can develop perforation while removing or cauterizing the granulations as they involve the fibrous layer of TM. In patients whom multiple types of sequelae $\left(\mathrm{S}_{2}\right.$ or $\mathrm{S}_{3}$ and/or $\left.\mathrm{S}_{4}\right)$ are present, all types of sequelae should be mentioned in the record; however, during staging, patients with the most severe type of sequela present should be considered. The GM patients with a small tiny perforation of TM should be differentiated from tubotympanic type of CSOM. In patients with GM, the small perforation of TM is surrounded or hidden by granulations and the middle ear mucosa is normal. Granulations/polyp arising from the middle ear mucosa in CSOM would be protruding through the perforation of TM. The untreated GM was found to cause post-inflammatory medial EAC fibrosis and its atresia. ${ }^{10}$

Similar to TNM classification and staging of cancer patients, on the basis of types of G, A and S lesions, GAS classification categorizes the patients with GM into four stages (I, II, III, and IV), which are further subdivided into three subgroups (a, b, and c). The Stage I-a is the mildest form of GM, while stage IV-c is the most severe form of GM. While classifying patients with GM, all types of G, A, and S lesions should be mentioned; however, during staging, the highest grade of $G$, $\mathrm{A}$, and $\mathrm{S}$ present should be considered.

\section{Example}

If a patient with GM has de-epithelization with sessile and pedunculated granulations, which involve almost entire TM with TM perforation, the GAS classification, and staging would be as following:

$$
\begin{aligned}
& \text { G: } G_{1}, G_{2}, G_{3} \\
& \text { A: } A_{3} \\
& \text { S: } S_{3} \\
& \text { Stage III-c: } G_{3}, A_{3}, S_{3}
\end{aligned}
$$

This complete classification and staging would provide the entire clinical and surgical findings of the patients with GM. The prior requirement of GAS classification and staging would be proper suction cleaning of the EAC under the operative microscope. GAS classification and staging could get altered after taking into consideration the surgical findings. The management would become more difficult as the stage advances; however, it needs to be confirmed by further studies. Patients with stages I and II (GM without TM perforation and granular otitis externa) are more common and relatively easy to manage with topical medicines than those with stages III and IV. Patients with stage III (GM with and without TM perforation), if do not respond with topical medication, would need surgery. Patients with Stage IV (GM with ear polyp, GM with granular otitis externa and GM with stenosis of EAC) are rare and comparatively difficult to manage and usually need surgical intervention. Patients with the last stage IV $\left(\mathrm{G}_{1-4}, \mathrm{~A}_{1-4}, \mathrm{~S}_{4}\right.$ - GM with stenosis of EAC) are the most challenging one.

\section{Conclusion}

The study proposes a novel GAS classification and staging system for patients with GM on the pattern of TNM oncological classification. It is simple and comprehensive and succinctly describes the lesions of GM and addresses prognosis and treatment decisions for patients with GM. GAS classification and staging system is based upon the types of Granulating lesion, Area of involvement, and Sequelae of the lesion. Patients are grouped into four stages, which indicate the overall severity and aids in speculating prognosis of the disease. The severity of disease influences outcomes in patients with GM. This novel classification should facilitate in selecting different modalities of treatment available and their outcome research in patients with GM. Each stage 
displays distinct features that would have substantial impact on decision making and prognosis. The pathology, management, and outcomes of each stage need to be assessed by further studies to evaluate the clinical and surgical relevance of this novel classification and staging for the patients with GM.

Conflict of Interest

None declared.

\section{Acknowledgment}

The literature and data sources were explored with the assistance of librarian Ms. Purvi Nirmal (MLiSc) of Gujarat Adani Institute of Medical Sciences. Discrepancies of data were discussed with Dr. Narendra Hirani (M.S.) and Dr. Chandrasekhar Ranade (M.S.), faculties in the E.N.T. department of Gujarat Adani Institute of Medical Sciences. Levels of evidence and other biostatistics matter were discussed with biostatistician Dr. Ajay Pathak (PhD) of the P.S.M department of P. S. Medical College.

\section{References}

1 Bansal M. Essentials of Ear, Nose and Throat. 1st ed. New Delhi, Jaypee Brothers Medical Publishers, 2016:85-94

2 Bansal M. Diseases of Ear, Nose and Throat. 2nd ed. New Delhi, Jaypee Brothers Medical Publishers, 2018:198-209

3 Bansal M. Granular myringitis: Current status of management. Int J Otorhinolaryngol Head Neck Surg 2015;1:55-57

4 Wolf M, Primov-Fever A, Barshack I, Polack-Charcon S, Kronenberg J. Granular myringitis: Incidence and clinical characteristics. Otol Neurotol 2006;27(8):1094-1097

$5 \mathrm{Kim}$ YH. Clinical characteristics of granular myringitis treated with castellani solution. Eur Arch Otorhinolaryngol 2011;268(8):1139-1146

6 Atef AM, Hamouda MM, Mohamed AH, Fattah AF. Topical 5-fluorouracil for granular myringitis: a double-blinded study. J Laryngol Otol 2010;124(3):279-284
7 Blevins NH, Karmody CS. Chronic myringitis: prevalence, presentation, and natural history. Otol Neurotol 2001;22(1):3-10

8 El-Seifi A, Fouad B. Granular myringitis: is it a surgical problem? Am J Otol 2000;21(4):462-467

9 Levi JR, Ames JA, Gitman L, Morlet T, O'Reilly RC. Clinical characteristics of pediatric granular myringitis. Otolaryngol Head Neck Surg 2013;148(2):291-296

10 Bansal M. Why Cannot We have an Etiological Classification for the Patients with Granular Myringitis? Indian J Otolaryngol Head Neck Surg 2017;69(3):397-400

11 Cheng YF, Shiao AS. Intractable chronic myringitis treated with carbon dioxide laser microsurgery. Arch Otolaryngol Head Neck Surg 2008;134(2):152-156

12 Jang $\mathrm{CH}$, Kim YH, Cho YB, Wang PC. Endoscopy-aided laser therapy for intractable granular myringitis. J Laryngol Otol 2006;120(7):553-555

13 Wolf M, Primov-Fever A, Barshack I, Kronenberg J. Granular myringitis in children. Int $\mathrm{J}$ Pediatr Otorhinolaryngol 2001;57(1):17-20

14 Zhang Z, Liu X, Chen S, Zheng Y. Combined tympanic epithelial layer avulsion and overlay myringoplasty for diffuse granular myringitis. J Laryngol Otol 2010;124(8):842-845

15 Hoshino T, Ueda Y, Mukohdaka H, Mizuta K. Acute granulomatous myringitis. J Laryngol Otol 1998;112(2):150-153

16 Boedts D. Myringitis granulosa. Acta Otorhinolaryngol Belg 1995;49(2):187-189

17 Fechner FP, Cunningham MJ, Eavey RD. Laser therapy for refractory myringitis in children. Otolaryngol Head Neck Surg 2002;127(3):163-168

18 Jung HH, Cho SD, Yoo CK, Lim HH, Chae SW. Vinegar treatment in the management of granular myringitis. J Laryngol Otol 2002;116(3):176-180

19 Yinglin Z. Three cases of chronic granular myringitis cured by formalin. J Otolaryngol 1985;14(6):379-380

20 Stoney P, Kwok P, Hawke M. Granular myringitis: a review. J Otolaryngol 1992;21(2):129-135

21 Makino K, Amatsu M, Kinishi M, Mohri M. The clinical features and pathogenesis of myringitis granulosa. Arch Otorhinolaryngol 1988;245(4):224-229 